https://helda.helsinki.fi

\title{
Lymphatic Vessels in Tumor Dissemination versus Immunotherapy
}

\section{Vaahtomeri, Kari}

2020-09-01

Vaahtomeri , K \& Alitalo , K 2020 , ' Lymphatic Vessels in Tumor Dissemination versus Immunotherapy ' , Cancer Research , vol. 80 , no. 17 , pp. 3463-3465 . https://doi.org/10.1158/0008-5472.CAN-20-0

http://hdl.handle.net/10138/331980

https://doi.org/10.1158/0008-5472.CAN-20-0156

unspecified

acceptedVersion

Downloaded from Helda, University of Helsinki institutional repository.

This is an electronic reprint of the original article.

This reprint may differ from the original in pagination and typographic detail.

Please cite the original version. 


\title{
Lymphatic vessels in tumor dissemination vs. immunotherapy
}

\author{
Kari Vaahtomeri ${ }^{1}$ and Kari Alitalo ${ }^{1,2, *}$ \\ ${ }^{1}$ Wihuri Research Institute and Translational Cancer Medicine Research Program, \\ University of Helsinki, Biomedicum Helsinki, Haartmaninkatu 8, 00290 Helsinki, \\ Finland \\ 2 iCAN Digital Precision Cancer Medicine Flagship, \\ University of Helsinki, Helsinki, Finland \\ *Corresponding author: \\ Kari Alitalo \\ Translational Cancer Medicine Program and Wihuri Research Institute \\ Biomedicum Helsinki, University of Helsinki \\ PO Box 63 (Haartmaninkatu 8), 00014 Helsinki, Finland \\ Phone: 3589-1912-5511 \\ E-mail: kari.alitalo@helsinki.fi \\ Fax: 3589-1912-5510 \\ Words (main text): 1925 (Epub ahead of print)
}

Abstract: 100

Refs: 14

Keywords: Tumor lymphangiogenesis, VEGF-C, metastasis, cancer immunotherapy Running title: Lymphatics in metastasis vs immunotherapy

The authors declare no potential conflict of interest. 


\begin{abstract}
During the growth of various cancers, primary tumors can escape anti-tumor immune responses of their host and eventually disseminate into distant organs. Peritumoral lymphatic vessels connect the primary tumor to lymph nodes, facilitating tumor entry into lymph nodes, systemic circulation, and metastasis. Lymph node metastases that occur frequently provide sites of tumor cell spread, whereas tumor antigen transfer into and presentation in tumor-draining lymph nodes induce activation of tumor-specific T-lymphocyte responses that can result in cytolytic targeting of the tumor. Here we discuss the recently emerged controversial role of the lymphatic vessels in tumor dissemination and cancer immunotherapy.
\end{abstract}

A hierarchy of lymphatic vessels that can transport metastatic cells to blood circulation traverses most tissues of our body. In its tree-like organization, the lymphatic capillaries at the top receive tissue fluid and cells, such as dendritic cells. Downstream of the capillaries, this fluid (now called lymph), and the immune cells, run into lymphatic collectors, where the surrounding smooth muscle cells assist flow into a series of lymph nodes. Whereas dendritic cells migrate into the lymph nodes to present their antigens to T-lymphocytes, thereby launching an adaptive immune response, the transported lymph is sieved through lymph node marginal sinus and trabecular conduits to enter efferent lymph vessels wherefrom lymph enters to blood circulation via the thoracic duct that connects to the subclavian vein. 
Historically, lymphatic vessels have been linked to lymph node metastasis, tumor dissemination, and poor prognosis of tumor patients. Production of lymphangiogenic growth factors, such as vascular endothelial growth factor C (VEGF-C), was shown to result in tumor lymphangiogenesis and lymphatic metastasis, and ablation of lymphatic vessels or inhibition of their growth reduced tumor dissemination in immune-compromised mice (1). More recently, lymph nodes were shown to provide sites of tumor cell entry into the blood circulation $(2,3)$.

However, Galon et al. drew attention to the finding that a combination of lymphatic vessels at the tumor margin and high immunoscore (i.e. the cytotoxic immune signature of the tumor) correlated with lack of metastases and improved patient survival in colorectal carcinoma (4). His contention has gained credit with recent advances in immunotherapy, which provided a possibility to ascertain mechanisms of how the lymphatic vessels in the tumor stroma of immunocompetent mice can promote anti-tumor immune responses via augmenting the activation of cytotoxic T-lymphocytes.

\section{TUMOR LYMPHANGIOGENESIS}

Lymphatic networks formed during embryonic and postnatal development retain the ability to expand when exposed to cytokines produced by tumor cells or inflammatory cells. The tumorigenic process affects the tumor-draining lymphatics in several ways, including lymphangiogenesis and dilation of lymphatic vessels, resulting in increased lymph transport, or sometimes by blocking lymph flow, directing lymph to collateral pathways of drainage. The expansion of lymphatic vessels is commonly caused by increased expression 
of VEGF-C by the tumor-associated leukocytes, especially by macrophages, or by the tumor cells themselves (1).

In several types of tumors, such as melanomas, lymphangiogenesis occurs mainly at the tumor margin and, to some extent, in tumor-draining lymph nodes. Lymphatic vessels at the tumor margin are capable of transporting immune cells and draining tissue fluid and their density correlates with patient survival in colorectal carcinoma (4), but they can also mediate lymphatic metastasis. Lymphatic vessels inside the tumor result from entrapment by the growing tumor or vessel invasion and they may contain intraluminal tumor cells. However, the growing primary tumor causes compression of the intratumoral lymphatic vessels, which leads to compromised flow of fluid and macromolecules and, thus, increased interstitial fluid pressure in the tumor (5). Thus, it is not yet clear, to what extent tumor cells or antigen-presenting cells in the intratumoral vessels migrate to the draining lymph nodes. Furthermore, the growth of tumor metastases in the lymph nodes may block lymphatic drainage, forcing a switch of lymphatic flow and metastasis to alternate lymph nodes.

\section{TUMOR DISSEMINATION VIA THE LYMPHATIC VESSELS}

Lymphatic vessel density at the tumor margin and lymph node metastases, especially in non-sentinel lymph nodes, has been considered to correlate with poor prognosis of patients with melanoma and breast, colorectal, and lung cancer. Removal of sentinel lymph nodes only incrementally improves patient prognosis. It has thus been questioned if lymph node metastases reflect only the overall metastatic capability of the primary tumor or if they 
present sites where tumor cells acquire further metastatic capabilities in immune-evasive conditions for their entry into the systemic circulation. Mouse tumor xenograft models in immunocompromised mice have indicated that tumor cell-produced lymphangiogenic factors enhance tumor metastasis, whereas blocking of VEGF-C/VEGFR3 signaling axis attenuates metastasis (1). A recent analysis of clonal tumor cell lineages in human colorectal cancers indicated that liver and lymph node metastases were derived from the same tumor cell subclones in one-third of the cases (6).

Afferent lymphatic vessels lead metastatic tumor cells into the lymph node subcapsular sinus, from where the tumor cells invade the lymph node stroma where they can enter the blood circulation via the high endothelial venules $(2,3)$. Alternatively, after passage through a series of lymph nodes, the tumor cells can migrate through the thoracic duct to enter the subclavian vein and blood circulation to seed organ metastasis.

\section{LYMPHATIC VESSELS SERVE ADAPTIVE ANTI-TUMOR IMMUNITY}

The involvement of lymphatic vessels in tumor immunity re-entered into focus only recently with the revival of the concept of tumor immunotherapy. Genetic damage in tumor cells creates tumor-antigens, some of which induce anti-tumor immune responses. Tumor neoantigens are presented in the lymph nodes by type 1 and type 2 conventional dendritic cells leading to priming of $\mathrm{CD} 8+$ and $\mathrm{CD} 4+\mathrm{T}$ cells, respectively and subsequent $\mathrm{T}$ lymphocyte mediated immunoediting of the tumor. Expansion and activation of type 1 conventional dendritic cells synergized with immune checkpoint inhibition by anti-PD-L1 antibodies in a mouse melanoma model $(7,8)$. Furthermore, as reported by Galon et al., 
lack of colorectal tumor metastases in patients correlated with a high lymphatic vessel density at the invasive tumor margins and with high CD8+ T-lymphocyte density in the tumor center (4). Conversely, the absence of lymphatic vessels was associated with reduced tumor leukocyte infiltration in a mouse melanoma model (9).

Besides the lymph nodes, the tumor stroma can also provide suitable conditions for Tlymphocyte priming at the primary tumor site. Chronic local tumor-induced inflammation can lead even to the formation of tertiary lymphoid organs, which are de novo assemblies resembling secondary lymphoid organs. Together with the lymphatic vessels, they modulate tumor microenvironment by expression of chemokines, such as CCL21, CXCL12, and CCL2, which act as guidance cues for naïve T-cells and antigen-presenting dendritic cells. Tertiary lymphoid organs act also as sites of T- and B-lymphocyte activation. In several tumor types, they correlate with CD4+ and CD8+ T-lymphocyte abundancy in tumor stroma and often, but not always, a favorable patient prognosis. In mouse tumor models, the priming of naïve and stimulation of active CD8+ T-lymphocytes in the primary tumor depends on type 1 conventional dendritic cells, which were necessary for the cytotoxic activity of adoptively transferred tumor-targeting T-lymphocytes (7).

Even in the presence of functional lymphatic drainage and anti-tumor T-lymphocyte priming, many tumors remain unresponsive to checkpoint blockade therapy. This can be due to a range of factors related to the quality of T-lymphocyte priming in the lymph node and to local T-lymphocyte suppression in the tumor microenvironment. These latter factors can include T-lymphocyte exclusion from the tumor core, down-regulation of major 
histocompatibility complex molecules, prevention of dendritic cell maturation, suppression by regulatory T-lymphocytes and direct inhibition by checkpoint proteins, such as PD1/PD-L1 (7).

As part of the stroma at tumor margin, also lymphatic endothelium can suppress anti-tumor responses, for example, by mechanisms such as inhibition of dendritic cell maturation via ICAM-MAC-1 interaction or inhibition of T-lymphocyte proliferation via expression of transforming growth factor-beta, indoleamine-2,3-dioxygenase or nitric oxide. Lymphatic endothelium may also promote tolerance towards tumor antigens by cross-presenting endogenous antigens to T-lymphocytes in the absence of co-stimulatory molecules or by suppressing the effector function of activated T-lymphocytes via their PD1 checkpoint receptor. Such mechanisms can result in the suppression of tumor-activated T-cells and their exhaustion $(1,10)$. Accordingly, the presence of lymphatic vessels was reported to cause local immune suppression of transferred cytotoxic T-lymphocytes in a mouse melanoma model (9).

Considering the multitude of ways by which lymphatic endothelia and other tumorassociated cells can promote antigen tolerance, it is remarkable that anti-CTLA4, by relieving the suppression of T-lymphocyte priming in the lymph nodes, and anti-PD1/PDL1 immunotherapy, by allowing execution of T-lymphocyte function within tumors, can promote anti-tumor responses, especially in melanoma (7). For successful checkpoint blockade immunotherapy, it is important that the lymphatic vessels traffic dendritic cells to the tumor-draining lymph node for the priming of T-lymphocytes. Well-functioning 
lymphatic vessels should thus be beneficial especially in tumors with a high mutational burden, for example after cytotoxic therapy, which produces mutations, tumor cell death, inflammation, and associated lymphangiogenesis, leading to increased antigen exposure to the immune system.

\section{CHEMOKINE CUES FOR LYMPH NODE HOMING/DISSEMINATION}

Dendritic cell homing to lymph nodes depends on chemokine receptor 7 (CCR7) and its CCL21 ligand, produced by lymphatic capillary endothelial cells in various tissues and in the lymph nodes, expression of the CCL19 ligand is restricted to lymph nodes. Occasionally, tumor cells can acquire CCR7 expression, promoting their directed migration towards lymphatics and invasion to lymph node parenchyma. Indeed, CCR7 expression by the tumor cells correlates with lymph node metastasis of gastric carcinoma, colorectal carcinoma, and breast cancer (1). Furthermore, VEGF-C produced by tumor cells increases CCL21 expression in nearby lymphatic endothelium, promoting a paracrine cross-talk that can foster lymph node metastasis (11). Recently, it was shown that the atypical chemokine receptor ACKR4 restrains antitumor immunity by decreasing intratumor CCL21. Deletion of ACKR4 in mice resulted in increased intratumor CCL21, retention of $\mathrm{CD} 103+$ dendritic cells, and an increase in $\mathrm{CD} 8+$ T-lymphocytes, which inhibited tumor growth (12). 


\section{VEGF-C BOOSTS IMMUNOTHERAPY IN MELANOMA AND GLIOMA}

\section{ISOGRAFTS}

Based on the recent findings, it thus seems that boosting lymphangiogenesis, antigen transport and lymphatic endothelial production of CCL21 chemokine by VEGF-C treatment should synergize with anti-tumor immunotherapy. Indeed, VEGF-C signaling and concomitant lymphangiogenesis enhanced the host response to an anti-tumor peptide vaccine and anti-PD1 immunotherapy in a mouse melanoma model (11). Furthermore, VEGF-C induced lymphangiogenesis enhanced T-cell priming and prevented, in a synergistic manner with anti-PD1 therapy, the growth of brain tumors in syngenic mouse models of glioma and melanoma (13). Gliomas, including their most aggressive form, glioblastoma, are genetically heterogeneous and less responsive to immunotherapy than many other cancers. Consistent with this, there is little evidence of immunoediting between initial and recurrent gliomas (14). VEGF-C could thus be useful in the treatment of gliomas in combination with cytotoxic or tumor vaccine therapy.

\section{OUTLOOK}

Lymphatics play a dual role in tumorigenesis: Whereas invasion of tumor cells through lymphatics to systemic circulation leads to metastases, the augmentation of T-cell activation via tumor antigen transport to lymph nodes is associated with a decreased metastatic burden and increased patient survival. Thus, boosting of lymphangiogenesis would be expected to lead to increased metastasis, but also improved priming of tumortargeting T-lymphocytes. Whether the net effect of tumor lymphatic vessels is metastasispromoting or boosting of anti-tumor immunity may depend on organ-specific differences 
in the lymphatic system in different anatomical sites. For example, meningeal lymphatic vessels appear to be rarely involved in tumor cell dissemination via the lymphatics, and instead of increasing metastasis, the VEGF-C stimulation of meningeal lymphangiogenesis seems to increase tumor antigen-mediated priming of $\mathrm{T}$ cells during immune checkpointtargeted therapy.

Further studies should highlight whether lymph nodes occupied by tumor cells can effectively function in T-lymphocyte priming and whether, despite lymph node metastases, an intact lymphatic system should be preserved to facilitate immunotherapy. An important question is if the improved immune response dominates upon eventual combination of lymphangiogenic factors with immune checkpoint inhibitors in human clinical trials. Due to the complex interplay between tumor/tumor stroma evolution and anti-tumor immunity, the planning of preclinical, and eventually clinical studies, should include evaluation of tumor location and stage, lymph node metastasis status, mutation load, immunoscore status, expression of CCR7 by the tumor cells, and lymphatic vessel density in the tumor area. Carefully designed studies should indicate if VEGF-C plus anti-tumor immunotherapy can be developed into personalized precision cancer medicine.

\section{ACKNOWLEDGMENTS}

We thank Dr. Ed Roberts (Beatson Institute, Glasgow) and Dr. Satu Mustjoki (University of Helsinki) for useful discussions and apologize to all those whose work could not be cited due to the space constraints. 
This work was funded by the Academy of Finland Research Fellow grant $(315710$, K. Vaahtomeri), University of Helsinki 3-year research grant (K. Vaahtomeri), Sigrid Juselius foundation young group leader grant (K. Vaahtomeri), iCAN Digital Precision Cancer Medicine Flagship (grant 320185, K. Alitalo), Academy of Finland (grants 292816, 273817, 312516 and 320249; K. Alitalo), Cancer Foundation Finland (K. Alitalo), Sigrid Juselius Foundation (K. Alitalo), Helsinki Institute of Life Sciences (HiLIFE, K. Alitalo), Biocenter Finland (K. Alitalo) and Hospital District of Helsinki and Uusimaa, Finland (K. Alitalo).

\section{REFERENCES}

1. Farnsworth RH, Achen MG, Stacker SA. The evolving role of lymphatics in cancer metastasis. Curr Opin Immunol. 2018;53:64-73.

2. Pereira ER, Kedrin D, Seano G, Gautier O, Meijer EFJ, Jones D, et al. Lymph node metastases can invade local blood vessels, exit the node, and colonize distant organs in mice. Science. 2018;359(6382):1403-7.

3. Brown M, Assen FP, Leithner A, Abe J, Schachner H, Asfour G, et al. Lymph node blood vessels provide exit routes for metastatic tumor cell dissemination in mice. Science. 2018;359(6382):1408-11.

4. Mlecnik B, Bindea G, Kirilovsky A, Angell HK, Obenauf AC, Tosolini M, et al. The tumor microenvironment and Immunoscore are critical determinants of dissemination to distant metastasis. Sci Transl Med. 2016;8(327):327ra26.

5. Padera TP, Stoll BR, Tooredman JB, Capen D, di Tomaso E, Jain RK. Pathology: cancer cells compress intratumour vessels. Nature. 2004;427(6976):695.

6. Naxerova K, Reiter JG, Brachtel E, Lennerz JK, van de Wetering M, Rowan A, et al. Origins of lymphatic and distant metastases in human colorectal cancer. Science. 2017;357(6346):55-60.

7. Binnewies M, Roberts EW, Kersten K, Chan V, Fearon DF, Merad M, et al. Understanding the tumor immune microenvironment (TIME) for effective therapy. Nat Med. 2018;24(5):541-50.

8. Perez CR, De Palma M. Engineering dendritic cell vaccines to improve cancer immunotherapy. Nat Commun. 2019;10(1):5408.

9. Lund AW, Wagner M, Fankhauser M, Steinskog ES, Broggi MA, Spranger S, et al. Lymphatic vessels regulate immune microenvironments in human and murine melanoma. J Clin Invest. 2016;126(9):3389-402. 
10. Card CM, Yu SS, Swartz MA. Emerging roles of lymphatic endothelium in regulating adaptive immunity. J Clin Invest. 2014;124(3):943-52.

11. Fankhauser M, Broggi MAS, Potin L, Bordry N, Jeanbart L, Lund AW, et al. Tumor lymphangiogenesis promotes $\mathrm{T}$ cell infiltration and potentiates immunotherapy in melanoma. Sci Transl Med. 2017;9(407).

12. Whyte CE, Osman M, Kara EE, Abbott C, Foeng J, McKenzie DR, et al. ACKR4 restrains antitumor immunity by regulating CCL21. J Exp Med. 2020;217(6).

13. Song E, T. Mao, H Dong, L.S.B. Boisserand, S. Antila, M. Bosenberg, K. Alitalo, J-L. Thomas and A. Iwasaki. VEGF-C-driven lymphatic drainage enables immunosurveillence of brain tumours. Nature. 2020.

14. Barthel FP, Johnson KC, Varn FS, Moskalik AD, Tanner G, Kocakavuk E, et al. Longitudinal molecular trajectories of diffuse glioma in adults. Nature. 2019;576(7785):112-20. 